\title{
Mortality of juvenile fishes of the genus Diplodus in protected and unprotected areas in the western Mediterranean Sea
}

\author{
E. Macpherson ${ }^{1, *}$, F. Biagi ${ }^{2}$, P. Francour ${ }^{3}$, A. García-Rubies ${ }^{1}$, J. Harmelin ${ }^{4}$, \\ M. Harmelin-Vivien ${ }^{4}$, J. Y. Jouvenel ${ }^{5}$, S. Planes ${ }^{5}$, L. Vigliola $^{4}$, L. Tunesi ${ }^{6}$ \\ ${ }^{1}$ Centro de Estudios Avanzados de Blanes (CSIC), Cami de Santa Barbara s/n, E-17300 Blanes, Girona, Spain \\ ${ }^{2}$ Dipartimento di Scienze dell'Uomo e dell'Ambiente, Universita di Pisa, Via A. Volta 6, I-5600 Pisa, Italy \\ ${ }^{3}$ GIS Posidonie, Parc Scientifique et Technique de Luminy, Case 901, F-13288 Marseille Cedex 09, France \\ ${ }^{4}$ Centre d'Océanologie de Marseille, CNRS UMR DIMAR, Station Marine d'Endoume, F-13007 Marseille, France \\ ${ }^{5}$ Ecole Pratique des Hautes Etudes, URA 1453 CNRS, Université de Perpignan, F-66860 Perpignan Cedex, France \\ ${ }^{6}$ ICRAM, Via L. Respighi 5, I-00197 Roma, Italy
}

\begin{abstract}
Mortality patterns from peak of settlement to dispersal from the nursery area, concurrently with integration of juveniles into adult populations, of 3 littoral fishes of the genus Diplodus ( $D$. puntazzo, D. sargus, and D. vulgaris) (Family Sparidae) were studied between May 1993 and June 1996. Twenty-one stations were censused weekly, weather conditions permitting, along the Mediterranean coasts of Spain. France, and Italy. Eight stations were located in protected areas and 13 in unprotected zones. Declines in abundance in all 3 species were particularly marked in the first month after settlement. Patterns of survivorship indicated that the 3 species were subject to density-dependent mortality from settlement to recruitment to the adult population. The effect of this mortality pattern was to reduce the variability in year-class strength, though without changing the rank order of abundance between years. The mortality rates for $D$. sargus were higher than for $D$. puntazzo and $D$. vulgaris. The results of this study indicated that mortality rates in protected areas did not differ significantly from those in unprotected zones, suggesting that marine reserves are not necessarily a sink for post-settlement fishes.
\end{abstract}

KEY WORDS: Mortality - Fish settlement - Diplodus - Marine reserves

\section{INTRODUCTION}

One of the main objectives of fish population research is to investigate the underlying causes determining the number of new individuals recruiting to the adult stage each year (year-class strength) (e.g. Shepherd \& Cushing 1990). Many authors have suggested that the variable and high mortality suffered by eggs and larvae during the planktonic stage is responsible for year-class strength (Cushing 1977). However, recent studies have shown that at least in certain groundfishes year-class strength may also be regulated during the post-settlement period by mortality in

\footnotetext{
•E-mail: macpherson@ceab.csic.es
}

juveniles that have settled to the bottom (e.g. Beverton \& Iles 1992b, Bailey 1994, Leggett \& Deblois 1994).

Most studies on juvenile mortality have been carried out on flatfishes (Zijlstra et al. 1982, van der Veer 1986, Tanaka et al. 1989, Iles \& Beverton 1991, van der Veer et al. 1991, Beverton \& Iles 1992a, b, Jager et al. 1995), gadoids (Sundby et al. 1989, Myers \& Cadigan 1993a, b, Tupper \& Boutilier 1995), rockfishes (Adams \& Howard 1996), gobies (Sano 1997), and on species mainly belonging to the Families Labridae and Pomacentridae in temperate and tropical waters (Victor 1986. Eckert 1987. Shulman \& Ogden 1987, Sale \& Ferrell 1988, Booth \& Beretta 1994, Levin 1994, Williams et al. 1994, Hixon \& Carr 1997). The results of such studies have shown that mortality rates may vary considerably and 
also that mortality may be either density-dependent (van der Veer 1986, Beverton \& Iles 1992b, Caley et al. 1996) or density-independent (Victor 1986, Doherty \& Williams 1988, Doherty \& Fowler 1994). As a result, analysis of mortality rates and comparison between species, areas, or years requires a thorough knowledge of the relationship between mortality and density (Bailey 1994, Caley et al. 1996, Hixon \& Carr 1997).

The causes of juvenile mortality are not yet clear (Bailey 1994, Leggett \& Deblois 1994), although certain studies have proved that predation may seriously affect mortality rates early in the benthic stage le.g. van der Veer \& Bergman 1987, Ellis \& Gibson 1995 , Connell 1997, Steele 1997), suggesting that the number of juveniles will increase when predators are excluded (Bailey \& Houde 1989, Carr \& Hixon 1995, Hixon \& Carr 1997). One consequence of these results is that juveniles in marine reserves, where potential predators are more abundant, may suffer a higher mortality rate, suggesting that these protected areas could act as a sink for post-settlement fishes (Roberts \& Polunin 1991). However, in spite of the interest in elucidating the effective role of marine reserves in the management of coastal fisheries (Bohnsack 1993, Carr \& Reed 1993), this relationship has not yet been studied.

The effects of protection on fish species have been discussed elsewhere (e.g. Roberts \& Polunin 1991 and references cited therein) and are quite evident, since fishing pressure is locally high and diverse in unprotected Mediterranean areas. There exist important differences in the fish assemblages inhabiting in protected and unprotected areas. The impact of protection, or the 'reserve effect', can be observed in a clear increase in species diversity, an increase in abundance and the average and maximum individual sizes of most target species, changes in the spatial distribution of many target species through recovery of shallowwater habitats from which they had been excluded by spearfishing (Bell. 1983, García-Rubies \& Zabala 1990, Harmelin et al. 1995) and alterations in the social conditions of the local populations of certain species (e.g. later induction of the sex change in Coris julis; Harmelin et al. 1995). These differences in fish assemblages can also have significant consequences for prey communities, e.g algae or sea urchins (Sala \& Zabala 1996, Sala \& Boudouresque 1997). Furthermore, some target species derive particular benefit from protection. Most of these species are carnivorous (e.g. Dentex dentex, Dicentrarchus labrax, Epinephelus marginatus, Scorpaena scrofa, Seriola dumerilii, Sciaena umbra, Sparus aurata), potentially large when adult, and especially threatened by highly selective and effective fishing, such as spearfishing. Other species particularly impacted by hook-and-line fishing are also carnivorous (e.g. Serranus cabrilla, S. scriba). All these species have much higher levels of density and/or biomass in protected areas, e.g. in the Medes Islands reserve fish biomass is more than 10 times higher than in the adjacent unprotected zone (Sala \& Zabala 1996 Garcia-Rubies 1997).

In the present study we have followed survivorship in 3 species of the genus Diplodus (D. puntazzo, D. sargus, and $D$. vulgaris) (Family Sparidae) from settlement to dispersal of juveniles out of the nursery areas and their recruitment to the adult population. The 3 species are common in the Mediterranean Sea, inhabiting littoral rocky bottoms and sea grass beds (Harmelin 1987, García-Rubies 1997). The settlement sites and settlement periods of these species are well known (García-Rubies \& Macpherson 1995, HarmelinVivien et al. 1995). Settlement of $D$. puntazzo and $D$. sargus takes place at very shallow depths ( 0 to $2 \mathrm{~m}$ ) on sandy-rocky bottoms in October-November and in May-June, respectively. The settlement period is usually very short (2 to $4 \mathrm{wk}$ ). In $D$. vulgaris settlement takes place on sandy bottoms over a broader depth range $(0$ to $7 \mathrm{~m}$ ), during a longer period (October to February), and there may be 2 distinct pulses (GarcíaRubies \& Macpherson 1995, Harmelin-Vivien et al. 1995). The settlement areas are situated at locations sheltered from the prevailing winds, usually bounded by promontories beyond which the coast is unprotected and exposed to wave action (García-Rubies \& Macpherson 1995, Harmelin-Vivien et al. 1995). On settlement, juveniles form a small number of monospecific shoals with a markedly clumped distribution. These monospecific shoals never mix with the shoals of adults present in the nursery area (Macpherson 1997). As the juveniles grow larger, the shoals fragment, presenting a less clumped distribution over the nursery area. The juveniles of the 3 species remain in the vicinity of the settlement sites for a number of months $(D$. puntazzo: 7 to $8 \mathrm{mo}$ D. sargus: 2 to $3 \mathrm{mo}$; D. vulgaris: 5 to $7 \mathrm{mo}$ ) until they reach 4.5 to $5.5 \mathrm{~cm}$ in length (Macpherson 1997). On attaining that size, they disperse outside the nursery area and join shoals of adult conspecifics normally measuring between 10 and $15 \mathrm{~cm}$ in size (Macpherson 1997).

The present study was carried out at 21 survey stations along the Mediterranean coasts of Spain, France, and Italy over a period of $3 \mathrm{yr}$ This sampling procedure was designed to ensure an accurate picture of mortality processes in these 3 species. Furthermore, 8 of the survey stations were located in marine protected areas and 13 in unprotected zones. The objectives of the study were to investigate whether the mortality rate on juveniles of these 3 species was density-dependent and whether the juveniles in marine reserves suffered higher rates of mortality than in unprotected areas. 


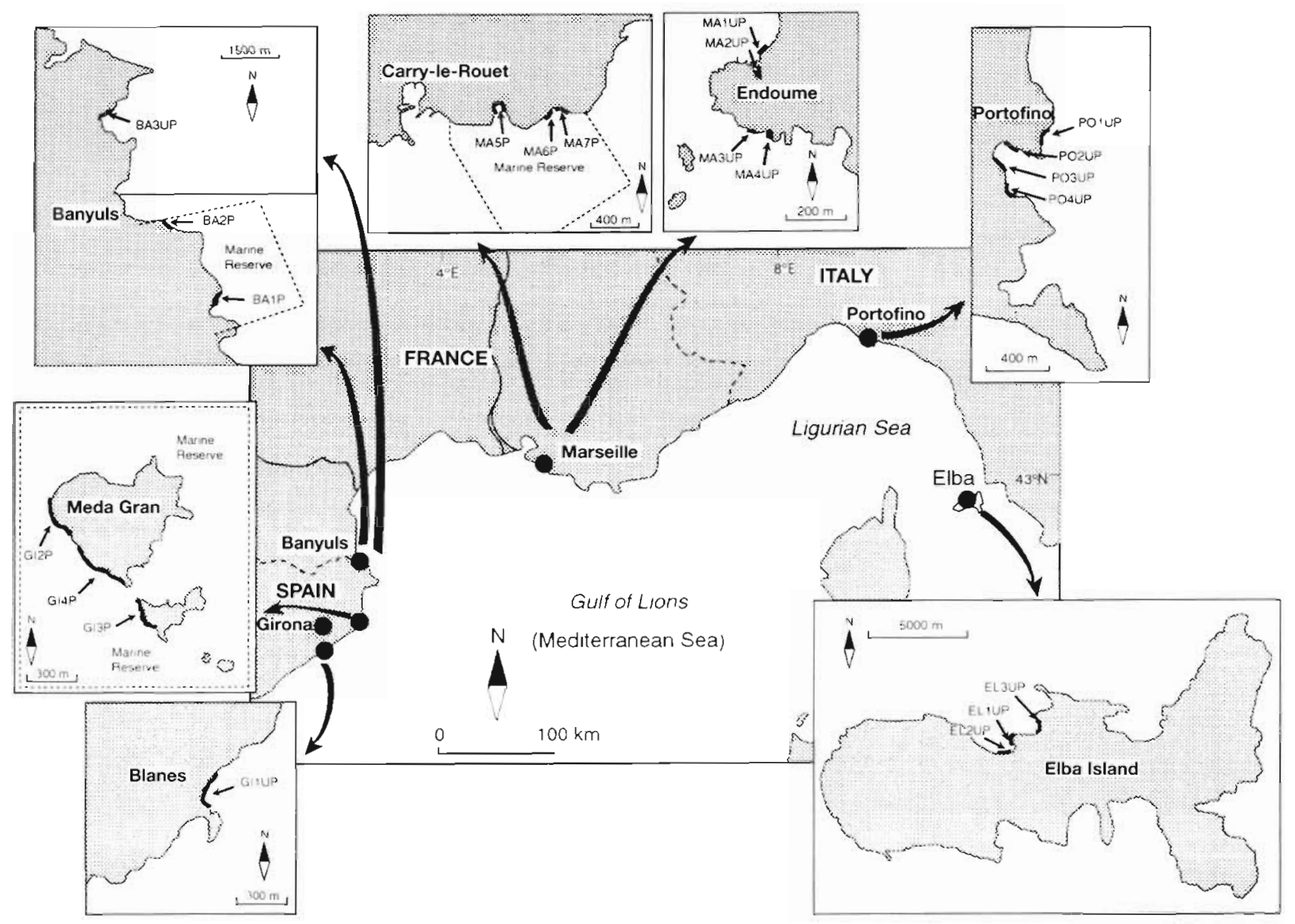

Fig 1 Map of the Northwestern Mediterranean Sea showing the location of sites used in this study Each site contains several sampling stations situated in small coves, in protected (P) and unprotected areas (UP) Stations were located in the coasts of Girona (GI, Spain), Banyuls (BA, France). Marselle (MA. France), Portotıno (PO, Italy) and Elba Island (EL, Italy)

\section{MATERIAL AND METHODS}

Data collection. Twenty-one stations were surveyed along the coasts of Spain (4 stations). France (10 stations) and Italy ( 7 stations). All of the stations were located in areas where settlement usually takes place, in small coves at very shallow depths $(<7 \mathrm{~m})$ on sandyrocky bottoms sheltered from the prevailing winds. The area surveyed by divers at each station was different, depending on the morphological characterıstıcs of the coast, and ranged between 27 and $650 \mathrm{~m}$ in length (Fig. 1). The stations situated in protected areas were located in Girona, Spain (GI2P, GI3P, GI4P), Banyuls, France (BA1P, BA2P) and Marselle, France (MA5P, MA6P, MA7PI The other stations were situated in unprotected zones.

Counts were made between May 1993 and June 1996. The sampling period spanned 3 settlement/postsettlement events for each species: Diplodus puntazzo (October to May); D. sargus (May to September); and
D. vulgaris (November to May). Nevertheless, at certaun stations only 2 events were sampled (Tables $1-3$ ). At all stations counts covered the whole station at depths between 0 and $7 \mathrm{~m}$, the depth range spanning the entire vertıcal distribution of settlers of these 3 species (Garcia-Rubles \& Macpherson 1995, Harmelin et al. 1995). New settlers of each of these 3 species, measuring 1.0 to $1.5 \mathrm{~cm}$ (total length), are readily distinguishable from those of the other 2 species. Settlers of $D$. puntazzo and $D$. sargus are practically unpıgmented except for the same dark vertical bands charactenstic of adults. Settlers of $D$. vulgans display even less pigmentation, and the 2 dark vertical bands characteristic of adults appear only after individuals have attanned a size of 2 to $3 \mathrm{~cm}$, several months after settlement. On each dive the observer swam slowly above the bottom from one end of the sampling site to the other, covernng the whole nursery area, counting all juveniles present and estimating their size. The duration of each dive was from $30 \mathrm{~min}$ (stations $<50 \mathrm{~m}$ in length) to $2 \mathrm{~h}$ (sta- 
tions $>300 \mathrm{~m}$ in length). Plastic tablets bearing silhouettes of individuals of different sizes were used to minimize errors in estimating specimen size (GarcíaRubies \& Macpherson 1995, Harmelin et al. 1995). Four replicates of each count were performed and the average taken as the true value. Furthermore, every instance of predation on the juveniles of each species was noted.

Weather conditions permitting, 1 or 2 dives were completed each week during the specific settlement periods for Diplodus puntazzo (October to December), $D$. sargus (May to June), and D. vulgaris (November to March) in order to obtain an accurate assessment of settlement abundance. After peak settlement abundance $\left(\mathbb{N}_{0}\right)$ had been recorded, censuses were carried out 1 to 4 times per month, depending on weather conditions, until dispersal of the post-settlers out of each nursery area. Recruitment to the adult population took place at the end of May-first half of June (Diplodus puntazzo and D. vulgaris) and in September (D. vulgaris) (Macpherson 1997). Thus, each time series of abundance used to estimate the mortality rate at each station in each year was calculated for the period running from the time of peak abundance of new settlers until dispersal of those same individuals. The behaviour of the juveniles of these Diplodus species (i.e. settlement in shallow water, no migration before dispersal; see Macpherson 1997) indicates that the loss of individuals can be attributed mainly to mortality, and in fact, censuses were no longer performed after individuals were observed outside the nursery areas. However, storms may affect the aggregation pattern of the shoals, and some unexpected fluctuations in abundance were observed at certain stations, especially in winter, when inclement weather conditions were more frequent (see below).

Data analysis. Applying the same methodology used in other studies on fish mortality (e.g. Vetter 1988, Iles \& Beverton 1991, Adams \& Howard 1996), individual abundance values for each census were $\ln (n+1)$ transformed and plotted on time (in d). The slope of the least-squares regression gave the instantaneous mortality rate $(M)$.

All Diplodus puntazzo and $D$. sargus settlers were considered to have arrived at the settlement area in a single arrival pulse, because these 2 species exhibit short settlement periods ( 2 to $4 \mathrm{wk}$ ). The short settlement period in both species produced a narrow range of size classes of juveniles at peak settlement, i.e. 1.0 to $2.0 \mathrm{~cm}$ (Macpherson 1997). D. vulgaris may exhibit 2 distinctly separate pulses, a smaller initial pulse (advance pulse) in November-December shortly before the main pulse (primary pulse) in JanuaryMarch, readily distinguishable by a clear difference in individual size, although individuals from the 2 pulses remained distinguishable over the study period at only a few stations (at the Blanes and Marseille stations). The advance pulse was not observed in all cases. Nevertheless, due to the low number of individuals observed during the advance pulse (peak abundance usually less than the $10 \%$ of the primary pulse), we have only considered the data from the primary pulse. The range of size classes at peak settlement in $D$. vulgaris was, however, wider than in $D$. puntazzo and $D$. sargus $(1.0$ to $2.5 \mathrm{~cm}$; Macpherson 1997).

In line with the procedure used by Iles \& Beverton (1991), time series that displayed fluctuations in abundance after peak settlement abundance not ascribable to sampling error in the censuses were not included in the data analysis (see 'Results'). These fluctuations were usually observed after storms affecting the nursery areas. Such unexpected increases/decreases were intcrpreted as migrations of individuals from/to other areas. Additionally, time series in which peak abundance was distinctly later than the time generally observed at most other localities were also excluded. Finally, series consisting of an insufficient number of samples during the settlement period were also omitted from the analysis.

The relationship between the mortality rate and peak settlement abundance was analysed using the Spearman rank-order correlation coefficient to establish whether the mortality pattern was densitydependent or density-independent. Furthermore, the action of density-dependent mortality in reducing inter-annual variation in year-class strength over the post-settlement stage was also analysed. To evaluate this effect the coefficient of variation (standard deviation divided by the mean, expressed as a percentage) for the peak settlement abundance $\left(N_{0}\right)$ values was compared with the coefficient of variation for the abundance value at the end of the post-settlement period just before dispersal $\left(\mathrm{N}_{t}\right)$. This coefficient is a good measure of the relative dispersion of the data (Zar 1984). A reduction in the coefficient between these 2 stages would provide confirmation that mortality is density-dependent (see also van der Veer 1986, Beverton \& Iles 1992b, Adams \& Howard 1996). Finally, the Mann-Whitney $U$-test was employed to compare the mortality values between species and in protected and unprotected areas by species.

\section{RESULTS}

Tables 1-3 present the results obtained for the different species, stations, and years. Analysis of the full data series showed that for Diplodus puntazzo 36 time 
Table 1. Diplodus puntazzo. Maximum abundances, mortality rates, and associated statistics. $T_{0}$ : date of peak abundance of new settlers; $N_{0}$ : maximum number of individuals during the settlement period; $M$ : slope of the regression equation $\left(d^{-1}\right)$; $N s:$ number of samples before dispersal used in mortality rate calculations; $\mathrm{N}_{t}$ : number of individuals at the end of the post-settlement period; $R^{2}$ : variation explained by the regression equation; SD: standard deviation. Station names were defined by site abbreviation (GI: Girona, Spain; BA: Banyuls, France; MA: Marseilles, France; PO: Portofino, Italy; EL: Elba, Italy), number ( 1 to 7$)$ and protected (P) and unprotected (UP) areas

\begin{tabular}{|c|c|c|c|c|c|c|c|c|c|c|}
\hline Site & Station & Year & $\mathrm{T}_{0}$ & $N_{0}$ & SD & $M$ & $\mathrm{SD}$ & Ns & $\mathrm{R}^{2}$ & $N_{1}$ \\
\hline \multirow[t]{9}{*}{ Girona } & GI1UP & 1993 & 23 Nov & 153 & 2.9 & 0.0120 & 0.0007 & 26 & 0.78 & 25 \\
\hline & GI1UP & 1994 & $17 \mathrm{Oct}$ & 340 & 17.8 & 0.0138 & 0.0010 & 24 & 0.60 & 27 \\
\hline & GI1UP & 1995 & 16 Nov & 26 & 0.0 & 0.0010 & 0.0006 & 18 & 0.82 & 7 \\
\hline & $\mathrm{GI} 2 \mathrm{P}$ & 1993 & $10 \mathrm{Oct}$ & 132 & 1.1 & 0.0069 & 0.0012 & 11 & 0.75 & 9 \\
\hline & $\mathrm{GI} 2 \mathrm{P}$ & 1994 & 23 Nov & 40 & 2.4 & 0.0047 & 0.0006 & 15 & 0.80 & 15 \\
\hline & GI3P & 1993 & 10 Nov & 36 & 1.5 & 0.0103 & 0.0013 & 17 & 0.86 & 3 \\
\hline & GI3P & 1994 & 24 Oct & 29 & 1.3 & 0.0086 & 0.0008 & 19 & 0.88 & 4 \\
\hline & GI4P & 1993 & 10 Nov & 134 & 0.0 & 0.0111 & 0.0010 & 14 & 0.91 & 17 \\
\hline & GI4P & 1994 & $24 \mathrm{Oct}$ & 114 & 0.0 & 0.0087 & 0.0007 & 15 & 0.92 & 11 \\
\hline \multirow[t]{6}{*}{ Banyuls } & BA1P & 1993 & $29 \mathrm{Dec}$ & 28 & 4.0 & - & - & - & - & - \\
\hline & BAlP & 1994 & $24 \mathrm{Oct}$ & 60 & 0.0 & 0.0051 & 0.0015 & 23 & 0.36 & 16 \\
\hline & $\mathrm{BA} 2 \mathrm{P}$ & 1993 & $23 \mathrm{Dec}$ & 31 & 5.0 & 0.0019 & 0.0022 & 15 & $<0.1$ & 13 \\
\hline & $\mathrm{BA} 2 \mathrm{P}$ & 1994 & 9 Nov & 26 & 0.0 & 0.0021 & 0.0014 & 21 & $<0.1$ & 10.5 \\
\hline & BA3UP & 1993 & $2 \mathrm{Dec}$ & 7 & 2.2 & 0.0026 & 0.0010 & 21 & 0.22 & 5 \\
\hline & BA3UP & 1994 & $24 \mathrm{Oct}$ & 35 & 0.0 & 0.0014 & 0.0002 & 27 & 0.67 & 2 \\
\hline \multirow[t]{18}{*}{ Marseille } & MA1UP & 1993 & 21 Nov & 30 & 1.2 & 0.0002 & 0.0015 & 18 & $<0.1$ & 19 \\
\hline & MA1UP & 1994 & 4 Nov & 8 & 0.5 & 0.0020 & 0.0001 & 19 & $<0.1$ & 7 \\
\hline & MA1UP & 1995 & 15 Nov & 18 & 0.0 & 0.0072 & 0.0024 & 14 & 0.41 & 3 \\
\hline & MA2UP & 1993 & 11 Feb $^{a}$ & 26 & - & - & - & - & - & - \\
\hline & MA2UP & 1994 & $15 \mathrm{Nov}$ & 3 & 0.01 & 0.0022 & 0.0019 & 18 & $<0.1$ & 2 \\
\hline & MA2UP & 1995 & $31 \mathrm{Oct}$ & 19 & 0.0 & 0.0010 & 0.0018 & 15 & 0.76 & 1 \\
\hline & MA3UP & 1993 & $14 \mathrm{Feb}^{\mathrm{a}}$ & 18 & - & - & - & - & - & - \\
\hline & MA3UP & 1994 & $15 \mathrm{Nov}$ & 2 & 1.0 & - & - & - & - & - \\
\hline & MA3UP & 1995 & $31 \mathrm{Oct}$ & 48 & 0.0 & 0.0070 & 0.0006 & 12 & 0.90 & 10 \\
\hline & MA4UP & 1993 & $28 \mathrm{Feb}^{\mathrm{d}}$ & 8 & - & - & - & - & - & - \\
\hline & MA4UP & 1994 & $1 \mathrm{Feb}^{d}$ & 2 & - & - & - & - & - & - \\
\hline & MA4UP & 1995 & $31 \mathrm{Oct}$ & 4 & 0 & 0.0023 & 0.0010 & 12 & 0.31 & 2 \\
\hline & MASP & 1993 & $6 \mathrm{Mar}^{\mathrm{a}}$ & 42 & - & - & - & - & - & - \\
\hline & MA5P & 1994 & $29 \mathrm{Oct}$ & 45 & 0.0 & 0.0084 & 0.0015 & 10 & 0.82 & 16 \\
\hline & MA6P & 1993 & $4 \mathrm{Dec}$ & 159 & 0.0 & 0.0102 & 0.0017 & 10 & 0.77 & 36 \\
\hline & MA6P & 1994 & $12 \mathrm{Dec}$ & 16 & 0.0 & 0.0074 & 0.0076 & 9 & 0.12 & 4 \\
\hline & MA7P & 1993 & $3 \mathrm{Mar}^{a}$ & 30 & - & - & - & - & - & - \\
\hline & MA7P & 1994 & $12 \mathrm{Dec}$ & 9 & - & - & - & - & - & - \\
\hline \multirow[t]{10}{*}{ Portofino } & POIUP & 1993 & $3 \mathrm{Dec}$ & 15 & 0.96 & 0.0015 & 0.0005 & 13 & 0.43 & 10 \\
\hline & PO1UP & 1994 & 13 Nov & 9 & 0.0 & 0.0026 & 0.0010 & 18 & 0.29 & 4 \\
\hline & PO1UP & 1995 & $20 \mathrm{Oct}$ & 3 & 0.0 & - & - & - & - & - \\
\hline & PO2UP & 1993 & $26 \mathrm{Nov}$ & 11 & 2.2 & 0.0006 & 0.0009 & 19 & $<0.1$ & 7 \\
\hline & PO2UP & 1994 & $2 \mathrm{DeC}$ & 23 & 0.0 & 0.0010 & 0.0030 & 14 & 0.45 & 3 \\
\hline & PO2UP & 1995 & 3 Nov & 6 & 0.0 & - & - & - & - & - \\
\hline & PO3UP & 1993 & $18 \mathrm{Mar}^{\mathrm{a}}$ & 8 & - & - & - & - & - & - \\
\hline & PO3UP & 1995 & 25 Nov & 11 & 0.0 & 0.0079 & 0.0008 & 13 & 0.94 & 3 \\
\hline & PO4UP & 1994 & 24 Nov & 28.9 & 0.1 & - & - & - & - & - \\
\hline & PO4UP & 1995 & $1 \mathrm{Dec}$ & 9 & 0.0 & 0.0028 & 0.0011 & 12 & 0.52 & 2.8 \\
\hline \multirow[t]{8}{*}{ Elba } & EL1UP & 1993 & $5 \mathrm{Dec}$ & 28 & 2.2 & 0.0060 & 0.0022 & 16 & 0.93 & 12 \\
\hline & EL1UP & 1994 & 2 Nov & 33 & 0.7 & 0.0059 & 0.0023 & 11 & 0.41 & 4 \\
\hline & EL2UP & 1993 & $15 \mathrm{Feb}^{\mathrm{a}}$ & 39 & - & - & - & - & - & - \\
\hline & EL2UP & 1994 & 1 Nov & 66 & 2.1 & 0.0095 & 0.0018 & 21 & 0.57 & 13 \\
\hline & EL2UP & 1995 & $10 \mathrm{Nov}$ & 80 & 1.4 & 0.0098 & 0.0011 & 30 & 0.87 & 4 \\
\hline & EL3UP & 1993 & $18 \mathrm{Jan}^{\mathrm{a}}$ & 34 & - & - & - & - & - & - \\
\hline & EL3UP & 1994 & 3 Nov & 253 & 26.9 & 0.0085 & 0.0028 & 14 & 0.44 & 12 \\
\hline & EL3UP & 1995 & $21 \mathrm{Oct}$ & 68 & 1.4 & 0.010 & 0.0009 & 24 & 0.92 & 3 \\
\hline
\end{tabular}


Table 2. Diplodus sargus. maximum abundances, mortality rates, and associated statistics. Abbreviations as in Table 1

\begin{tabular}{|c|c|c|c|c|c|c|c|c|c|c|}
\hline Site & Station & Year & $T_{0}$ & $N_{0}$ & SD & $M$ & SD & Ns & $\mathrm{R}^{2}$ & $N_{i}$ \\
\hline \multirow[t]{10}{*}{ Girona } & GI1UP & 1994 & $20 \mathrm{Jun}$ & 336 & 1.7 & 0.0195 & 0.0016 & 8 & 0.96 & 129 \\
\hline & GI1UP & 1995 & $14 \mathrm{Jul}$ & 223 & 1.0 & 0.0108 & 0.0019 & 9 & 0.81 & 133 \\
\hline & GI2P & 1993 & $14 \mathrm{Jul}$ & 44 & 3.2 & 0.0116 & 0.0010 & 7 & 0.96 & 19 \\
\hline & GI2P & 1994 & 8 Jun & 1051 & 136.3 & 0.0373 & 0.0040 & 9 & 0.93 & 84 \\
\hline & Gl2P & 1995 & $13 \mathrm{Jul}$ & 105 & 7.8 & 0.0234 & 0.0020 & 6 & 0.97 & 29 \\
\hline & GI3P & 1993 & $8 \mathrm{Jul}$ & 16 & 0.5 & 0.0128 & 0.0019 & 7 & 0.91 & 9 \\
\hline & GI3P & 1994 & $21 \mathrm{Jun}$ & 653 & 7.8 & 0.0185 & 0.0027 & 8 & 0.89 & 232 \\
\hline & GI3P & 1995 & $4 \mathrm{Jul}$ & 160 & 3.7 & 0.0110 & 0.0013 & 7 & 0.88 & 92 \\
\hline & GI4P & 1994 & 8 Jun & 1594 & 0.0 & 0.0322 & 0.0073 & 5 & 0.87 & 174 \\
\hline & GI4P & 1995 & $13 \mathrm{Jul}$ & 274 & 0.0 & 0.0101 & 0.0035 & 6 & 0.68 & 129 \\
\hline \multirow[t]{6}{*}{ Banyuls } & $\mathrm{BA} 1 \mathrm{P}$ & 1994 & $30 \mathrm{Jun}$ & 345.5 & 30.2 & 0.0300 & 0.0082 & 9 & 0.66 & 111 \\
\hline & BA.1P & 1995 & $4 \mathrm{Jul}$ & 64.6 & 2.6 & 0.0063 & 0.0054 & 7 & 0.21 & 31 \\
\hline & $\mathrm{BA} 2 \mathrm{P}$ & 1994 & 28 Jun & 183.2 & 7.3 & 0.0271 & 0.0067 & 8 & 0.73 & 27 \\
\hline & $\mathrm{BA} 2 \mathrm{P}$ & 1995 & 4 Jul & 28.8 & 11.5 & 0.0016 & 0.0061 & 6 & $<0.1$ & 27 \\
\hline & BA3UP & 1994 & $21 \mathrm{Jun}$ & 60 & 4.1 & 0.0111 & 0.0038 & 8 & 0.59 & 31 \\
\hline & BA3UP & 1995 & $6 \mathrm{Jul}$ & 55 & 2.6 & 0.0134 & 0.0057 & 7 & 0.52 & 37 \\
\hline \multirow[t]{15}{*}{ Marseille } & MA1UP & 1993 & $14 \mathrm{Jul}$ & 49 & 2.49 & 0.0118 & 0.0058 & 7 & 0.45 & 16 \\
\hline & MA1UP & 1994 & 15 Jun & 139 & 11.3 & 0.0080 & 0.0063 & 13 & 0.69 & 70 \\
\hline & MA1UP & 1995 & 26 Jun & 92 & 7.1 & 0.0161 & 0.0025 & 7 & 0.89 & 40 \\
\hline & MA2UP & 1994 & $15 \mathrm{Jun}$ & 171 & 43.9 & 0.0329 & 0.0037 & 6 & 0.95 & 29 \\
\hline & MA2UP & 1995 & 6 Jun & 117 & 0.0 & 0.0340 & 0.0060 & 7 & 0.86 & 27 \\
\hline & MA3UP & 1993 & $7 \mathrm{Jun}$ & 55 & 4.0 & 0.0005 & 0.0041 & 12 & $<0.1$ & 4.3 \\
\hline & MA3UP & 1994 & 8 Jun & 427 & 19.6 & 0.0241 & 0.0040 & 7 & 0.88 & 125 \\
\hline & MA3UP & 1995 & 19 Jun & 196 & 8.6 & 0.0245 & 0.0029 & 7 & 0.93 & 53 \\
\hline & MA4UP & 1993 & $21 \mathrm{Jun}$ & 47 & 8.3 & 0.0049 & 0.0011 & 13 & 0.68 & 35 \\
\hline & MA4UP & 1994 & 8 Jun & 346 & 43.8 & 0.0216 & 0.0024 & 10 & 0.93 & 124 \\
\hline & MA4UP & 1995 & 15 Jun & 165 & 4.0 & 0.0176 & 0.0056 & 10 & 0.62 & 59 \\
\hline & MASP & 1994 & 17 Jun & 703 & 0.0 & 0.0124 & 0.0028 & 6 & 0.83 & 414 \\
\hline & MA5P & 1995 & $15 \mathrm{Jul}$ & 173 & 0.0 & - & - & - & - & - \\
\hline & MA6P & 1994 & $17 \mathrm{Jun}$ & 165 & 0.0 & 0.0213 & 0.0048 & 5 & 0.87 & 41 \\
\hline & MA7P & 1994 & 24 Jun & 280 & 0.0 & 0.0216 & 0.0080 & 5 & 0.71 & 63 \\
\hline \multirow[t]{5}{*}{ Portofino } & PO1UP & 1994 & 27 Jun & 135 & 2.22 & 0.0183 & 0.0122 & 6 & 0.36 & 28 \\
\hline & PO2UP & 1994 & 9 Jun & 165 & 3.77 & 0.0317 & 0.0089 & 6 & 0.76 & 40 \\
\hline & PO2UP & 1995 & 28 Jun & 62 & 1.89 & 0.0231 & 0.0050 & 9 & 0.75 & 1.7 \\
\hline & PO4UP & 1994 & 27 Jun & 405 & 60.1 & 0.0395 & 0.0231 & 6 & 0.62 & 40 \\
\hline & PO4UP & 1995 & $14 \mathrm{Jul}$ & 60 & 8.35 & - & - & - & - & - \\
\hline \multirow[t]{6}{*}{ Elba } & EL1UP & 1994 & $12 \mathrm{JuI}$ & 86 & 0.0 & 0.0126 & 0.0037 & 5 & 0.80 & 40 \\
\hline & EL1UP & 1995 & $22 \mathrm{Jul}$ & 58 & - & - & - & - & - & - \\
\hline & EL2UP & 1994 & 19 Jun & 362 & 38.7 & 0.0255 & 0.0063 & 9 & 0.70 & 101. \\
\hline & EL2UP & 1995 & $17 \mathrm{Jul}$ & 50 & - & - & - & - & - & - \\
\hline & EL3UP & 1994 & 31 May & 330 & 16.3 & 0.0240 & 0.0063 & 7 & 0.74 & 82 \\
\hline & EL3UP & 1995 & 7 Jun & 84 & - & - & - & - & - & - \\
\hline
\end{tabular}

series of abundance (out of a total of 51) yielded adequate time series trends. A similar high proportion was obtained for $D$. sargus (37 time series out of a total of 42), and D. vulgaris (25 time series out of a total of 37). A subset of the density time series, showing those stations where sampling was performed in all $3 \mathrm{yr}$ and/or a wide range of values for the maximum number of individuals during the settlement period are represented graphically in Figs. 2-4. Standard deviation from the 4 replicates of each count were, in most cases, very low $(<5 \%$ of the mean, see also Tables $1-3$ ). For simplicity these values were not indicated in Figs. 2-4.

\section{Diplodus puntazzo}

Mortality rates $(M)$ spanned a wide range of values $\left(0.0006\right.$ to $\left.0.015 \mathrm{~d}^{-1}\right)$ (Table 1 ). At those stations where 2 time series were collected, the value of $M$ was lower when initial density was lower. The observed relationship between $N_{0}$ and $M$ was significant and positive for both periods (Spearman test: $\mathrm{n}=36, \mathrm{R}=0.67, \mathrm{p}<$ $0.0001)$, suggesting that mortality was clearly densitydependent. Density of survivors is an exponential function of time and accordingly the largest decrease was observed in the first month after settlement. This decrease was especially important $(>80 \%)$ in data 
Table 3. Diplodus vulgaris. Maximum abundances, mortality rates, and associated statistics. Abbreviations as in Table 1

\begin{tabular}{|c|c|c|c|c|c|c|c|c|c|c|}
\hline Site & Station & Year & $T_{0}$ & $N_{0}$ & SD & $M$ & $\mathrm{SD}$ & $\mathrm{Ns}$ & $\mathrm{R}^{2}$ & $N_{i}$ \\
\hline \multirow[t]{3}{*}{ Gerona } & GI1UP & 1994 & 5 Jan & 1775 & 34.5 & 0.0090 & 0.0014 & 13 & 0.65 & 439 \\
\hline & GI1UP & 1995 & $30 \mathrm{Jan}$ & 1812 & 28.6 & 0.0110 & 0.0009 & 14 & 0.88 & 507 \\
\hline & GI1UP & 1996 & 4 Jan & 1061 & 78.3 & 0.0090 & 0.0017 & 14 & 0.31 & 400 \\
\hline \multirow[t]{6}{*}{ Banyuls } & BA1P & 1994 & $16 \mathrm{Apr}$ & 436 & 0.0 & - & - & - & - & - \\
\hline & $\mathrm{BA} 1 \mathrm{P}$ & 1995 & 29 Feb & 36 & 0.0 & - & - & - & - & - \\
\hline & $\mathrm{BA} 2 \mathrm{P}$ & 1994 & 3 Mar & 490 & 35.4 & 0.0125 & 0.0021 & 9 & 0.81 & 122 \\
\hline & $\mathrm{BA} 2 \mathrm{P}$ & 1995 & $25 \mathrm{Jan}$ & 52 & 32.3 & - & - & - & - & - \\
\hline & BA3UP & 1994 & $16 \mathrm{Mar}$ & 795.5 & 38.9 & 0.0174 & 0.0029 & 8 & 0.83 & 129 \\
\hline & BA3UP & 1995 & $17 \mathrm{Mar}$ & 116 & 30.4 & - & - & - & - & - \\
\hline \multirow[t]{11}{*}{ Marseille } & MA1UP & 1994 & 17 Feb & 174 & 7.5 & 00045 & 0.0009 & 9 & 076 & 107 \\
\hline & MA1UP & 1995 & $17 \mathrm{Jan}$ & 3330 & 452 & 0.0136 & 0.0040 & 9 & 0.75 & 109 \\
\hline & MA1UP & 1996 & $19 \mathrm{Dec}$ & 678 & 12.5 & 0.0123 & 0.0026 & 7 & 0.70 & 34 \\
\hline & MA2UP & 1994 & 18 Feb & 178 & 19.5 & 0.0025 & 0.0032 & 9 & $<0.1$ & 125 \\
\hline & MA2UP & 1995 & $17 \mathrm{Jan}$ & 3151 & 500 & 0.0260 & 0.0045 & 10 & 0.81 & 112 \\
\hline & MA2UP & 1996 & $17 \mathrm{Jan}$ & 359 & 11.5 & 0.0082 & 0.0043 & 9 & 0.34 & 65 \\
\hline & MA3UP & 1995 & 1 Feb & 395 & 14.2 & 0.0135 & 0.0024 & 8 & 0.83 & 69 \\
\hline & MA3UP & 1996 & 15 Nov & 61 & 0.0 & - & - & - & - & - \\
\hline & MA4UP & 1995 & $16 \mathrm{Jan}$ & 2063 & 500 & 0.0279 & 0.0094 & 8 & 0.59 & 76 \\
\hline & MA4UP & 1996 & $21 \mathrm{Dec}$ & 42 & 0.02 & 0.0011 & 0.0051 & 8 & $<0.1$ & 15 \\
\hline & MA7P & 1994 & 3 Mar & 424 & 0.0 & 0.0324 & 0.0028 & 7 & 0.97 & 13 \\
\hline \multirow[t]{9}{*}{ Portofino } & PO1UP & 1994 & $18 \mathrm{Feb}$ & 57 & 5.1 & 0.0140 & 0.0044 & 11 & 0.83 & 12 \\
\hline & POIUP & 1995 & 4 Feb & 25 & 11.8 & 0.0089 & 0.0026 & 8 & 0.66 & 13 \\
\hline & PO1UP & 1996 & $14 \mathrm{Jan}$ & 17.3 & 0.5 & - & - & - & - & - \\
\hline & PO2LP & 1994 & 11 Feb & 80.8 & 2.4 & 0.0150 & 0.0023 & 12 & 0.94 & 12 \\
\hline & PO2UP & 1995 & 31 MaI & 128 & 16.7 & - & - & - & - & \\
\hline & PO2UP & 1996 & $12 \mathrm{Apr}$ & 82.5 & 4.0 & - & - & - & - & - \\
\hline & PO3UP & 1995 & 14 Apr & 219 & 0.0 & - & - & - & - & - \\
\hline & PO3UP & 1996 & $26 \mathrm{Apr}$ & 50.3 & 1.7 & - & - & - & - & - \\
\hline & PO4UP & 1996 & 19 Apr & 84.8 & 7.9 & - & - & -- & - & - \\
\hline \multirow[t]{8}{*}{ Elba } & EL1UP & 1994 & 14 Feb & 159 & 0.0 & - & - & - & - & - \\
\hline & EL1UP & 1995 & $17 \mathrm{Jan}$ & 112 & 10.4 & 0.0089 & 0.0031 & 7 & 0.63 & 50 \\
\hline & EL2UP & 1994 & 15 Feb & 533 & 0.0 & 00224 & 0.0047 & 10 & 0.68 & 90 \\
\hline & EL2UP & 1995 & $18 \mathrm{Jan}$ & 310 & 7.5 & 0.0046 & 0.0016 & 12 & 0.35 & 107 \\
\hline & EL2UP & 1996 & $6 \mathrm{Mar}$ & 180.5 & 10.6 & 0.0205 & 0.0044 & 14 & 0.64 & 23 \\
\hline & EL3UP & 1994 & 19 Jan & 459 & 41.0 & 0.0236 & 0.0027 & 12 & 0.88 & 32 \\
\hline & EL3UP & 1995 & $17 \mathrm{Mar}$ & 902 & 30.4 & 0.0244 & 0.0048 & 11 & 0.72 & 60 \\
\hline & EL3UP & 1996 & 12 Jan & 552 & 15.6 & 0.0193 & 0.0031 & 14 & 0.76 & 46 \\
\hline
\end{tabular}

series for large numbers of individuals (>200) (Fig. 2). The mortality trend was appreciably lower at lower abundance levels.

\section{Diplodus sargus}

The results recorded for Diplodus sargus followed a pattern similar to that observed in the preceding species. Mortality rates exhibited a certain variability between sites, stations, and years, ranging between 0.0005 and $0.034 \mathrm{~d}^{-1}$ (Table 2). The mortality rate increased with maximum settlement abundance in nearly all cases. Despite few exceptions, comparison of all the values of $M$ and $N_{0}$ yielded a significant and positive relationship between these 2 variables (Spearman test: $\mathrm{n}=38, \mathrm{R}=0.59, \mathrm{p}=0.0001$ ). Abundance trends over the post-settlement period exhibited a pattern similar to that observed in D. puntazzo. Again, the largest decrease was recorded during the first month after settlement (Fig. 3).

\section{Diplodus vulgaris}

Mortality rate estimates were also highly variable ranging between 0.001 and $0.032 \mathrm{~d}^{-1}$ (Table 3). As in the 2 preceding species, the mortality rate increased with peak settlement abundance. The relationship between $M$ and $N_{0}$ was again significant and positive (Spearman test: $\mathrm{n}=36, \mathrm{R}=0.50, \mathrm{p}<0.02$ ). Dipiodus vulgaris also underwent a major decrease in abundance during the first month after settlement (Fig. 4). 

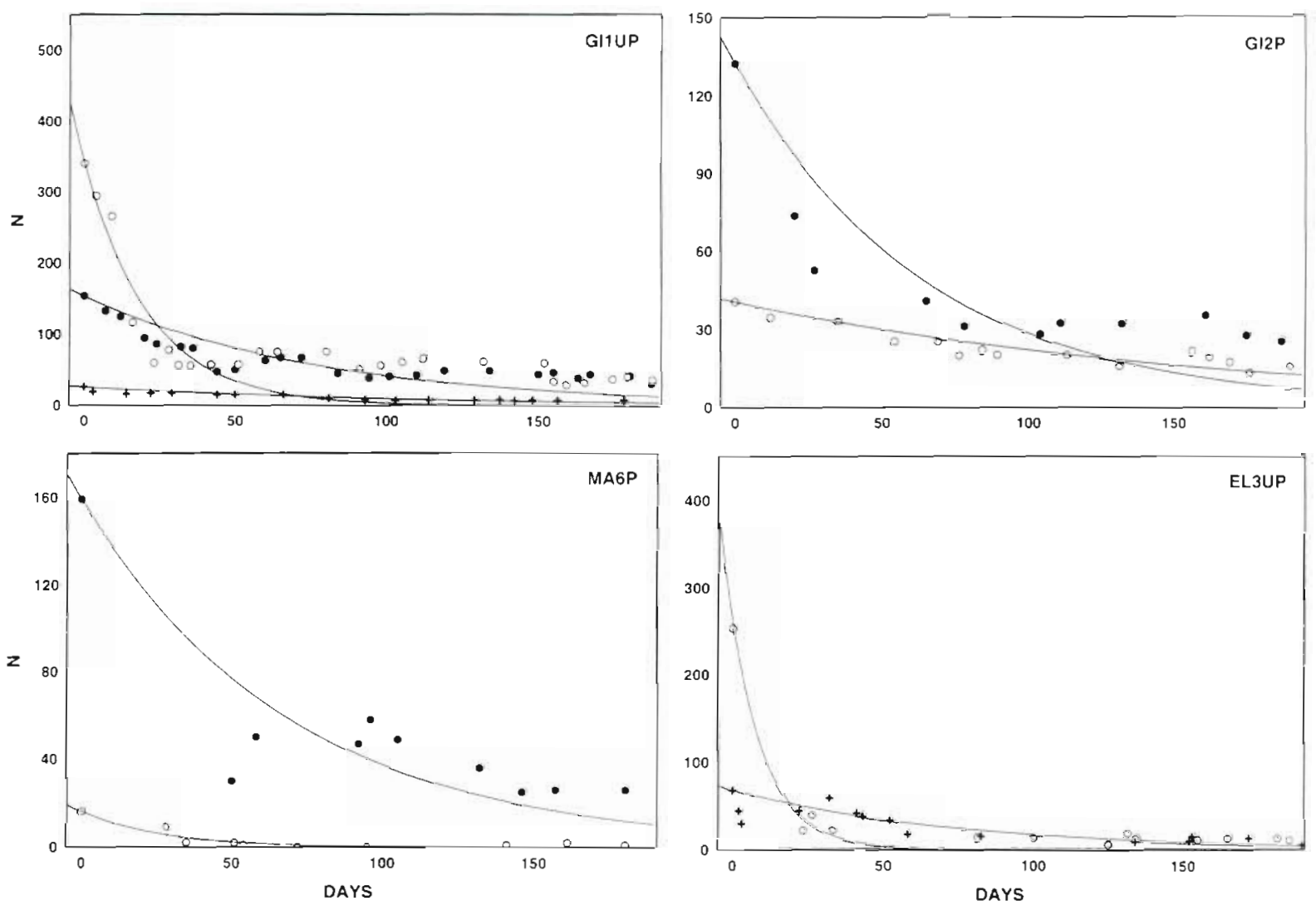

Fig. 2. Diplodus puntazzo. Density time series after peak settlement abundance. N: number of individuals (see text for further explanation). 0: date of maximum abundance at settlement. Years: $(\bullet)$ 1993, (0) 1994, (+) 1995. Stations were located in Girona (GI), Marseille (MA) and Elba Island (EL), in protected (P) and unprotected (UP) zones (see also Table 1)

\section{Interspecific differences in mortality rates}

Mortality rates over the post-settlement period were compared between species by grouping the different data series into 3 peak settlement abundance levels: low $(<100$ ind.), moderate (100 to 400 ind.) and high ( $>400$ ind.) to minimize the effect of density on the compared mortality values. Mortality rates for Diplodus sargus were higher than those for $D$. puntazzo (Mann-Whitney $U$-test: $p<0.05$ at low abundance, $p<0.01$ at moderate abundance; no comparisons were made at high abundance due to the low settlement level of $D$. puntazzo). The differences between $D$. sargus and $D$. vulgaris were not significant, except at the medium abundance level ( $D$. sargus was higher than $D$. vulgaris, $\mathrm{p}<0.01$ ). Mortality rates for $D$. puntazzo were only significantly lower $(\mathrm{p}<0.01)$ from those for $D$. vulgaris at the low abundance level.

\section{Reduced variability in year-class strength}

The coefficient of variation for the number of individuals, calculated from Tables $1-3$, was higher at set- tlement $\left(\mathrm{N}_{0}\right)$ than at the end of post-settlement $\left(\mathrm{N}_{t}\right)$. The reduction in the coefficient of variation was quite evident for Diplodus puntazzo and D. sargus. In $D$. puntazzo the coefficient of variation declined from ca $125 \%$ at settlement to ca $73 \%$ at the end of post-settlement. The trend was similar in D. sargus (ca $117 \%$ at settlement and ca $88 \%$ just before dispersalj. However, there was no reduction in the coefficient of variation for $D$. vulgaris, with the coefficient of variation remaining constant at ca 116 to ca $118 \%$.

\section{Mortality rates in protected and unprotected areas}

The values of $M$ calculated at stations located in protected and unprotected areas were compared at 3 different levels of settlement abundance. The 3 levels $(<100,100$ to 400 , and $>400$ ind.) were used to minimize the effect of density-dependent mortality patterns on the comparisons. The comparisons were made both for the sampling period as a whole and for each individual year. Mortality values were compared only for Diplodus puntazzo and D. sargus. This analysis was 

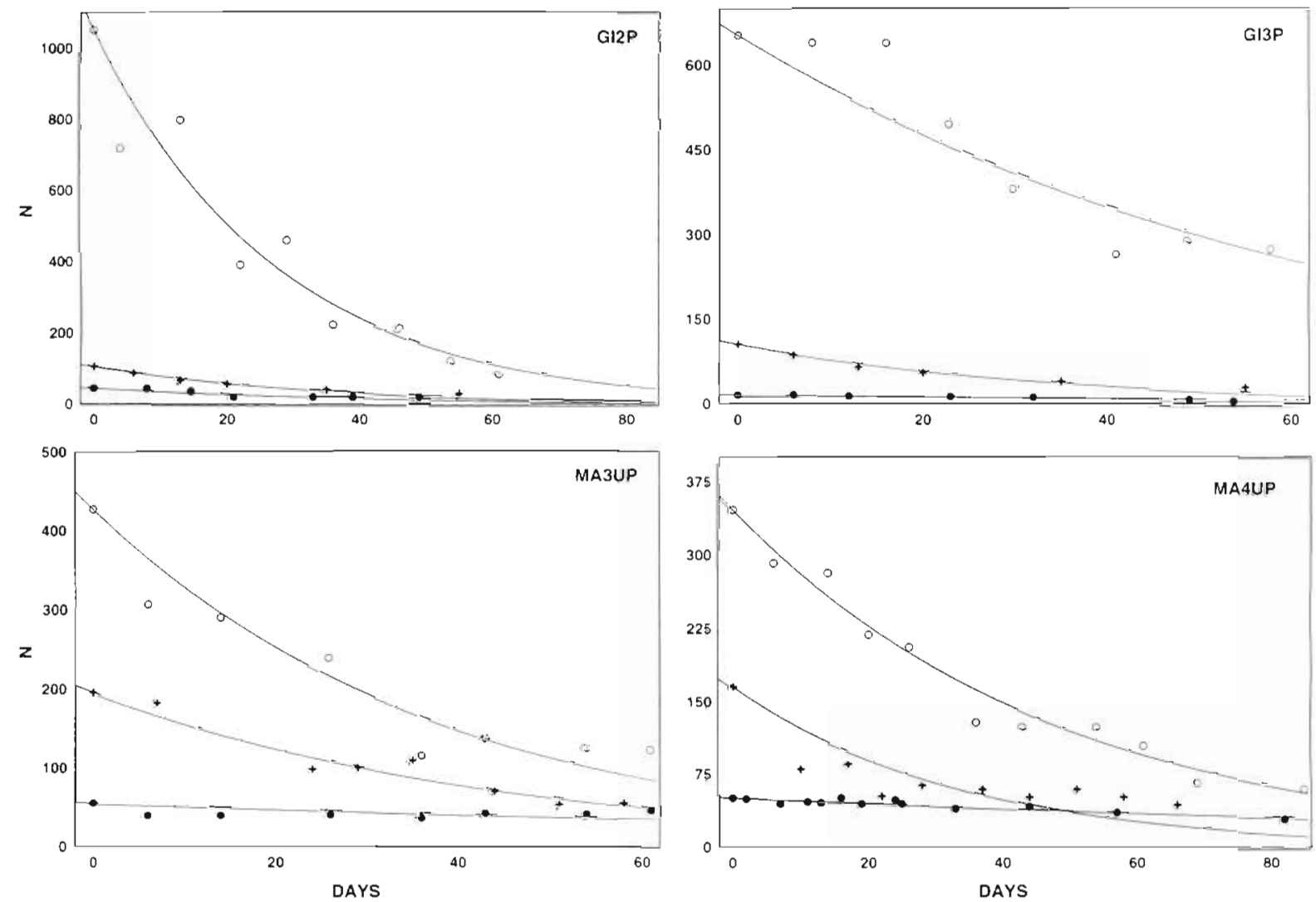

Fig. 3. Dipludus sargus. Density time series after peak settlement abundance. $N$ : number of individuals (see text for further explanation). 0: date of maxi-mum abundance at settlement. Years: ( $1993,(0) 1994,(+)$ 1995. Station abbreviations as in Fig. 2

not performed for $D$. vulgaris because of the small number of data series available for stations in protected areas. Neither species showed significant differences between the protected and unprotected areas at any of the 3 abundance levels, and the Mann-Whitney $U$-test was always non-significant (Table 4).

\section{DISCUSSION}

The results of the present study indicated that the 3 species of Diplodus (D. puntazzo, D. sargus, and $D$. vulgaris) were subject to density-dependent mortality from the onset of settlement to recruitment to the adult population. This pattern was especially evident in $D$. puntazzo and $D$. sargus but was less distinct in D. vulgaris. The reduction in the density of individuals was particularly marked in the first month after settlement; after that period mortality rates were clearly lower. In any case, the density-dependent mortality factor played a major role in determining the shape of the mortality curves. Accordingly, observed declines in abundance were high for time series in which settlement abundance was high, whereas declines were very low at low initial numbers of individuals.

Studies dealing with density-dependent patterns of juvenile mortality are hampered by a number of conceptual and methodological difficulties, and as a consequence some results are ambiguous and conclusions are not robust (Jones 1991, Bailey 1994). To date, 2 methods have been used: (1) density time series; and (2) regression of $\ln \left(N_{t} / N_{0}\right)$ versus time (mortality rate) (e.g. Victor 1986, Shulman \& Ogden 1987, Beverton \& Iles 1992b, Bailey 1994). This latter method may involve numerous statistical difficulties, particularly when the relationship between $\mathrm{N}_{t}$ and $\mathrm{N}_{0}$ values is weak (Dempster \& Pollard 1986, Hassell 1986, Gaston \& Lawton 1987, Hanski 1990, Hanski et al. 1993, but see Dennis \& Taper 1994). The density time series method, as it has been used in the present study, avoids these statistical problems and is perhaps the more accurate method for estimating mortality rates and evaluating the presence of density-dependent mortality processes (Bailey 1994).

The density time series method has not been commonly employed, mainly because it requires intensive sampling of the nursery area during the post-settle- 

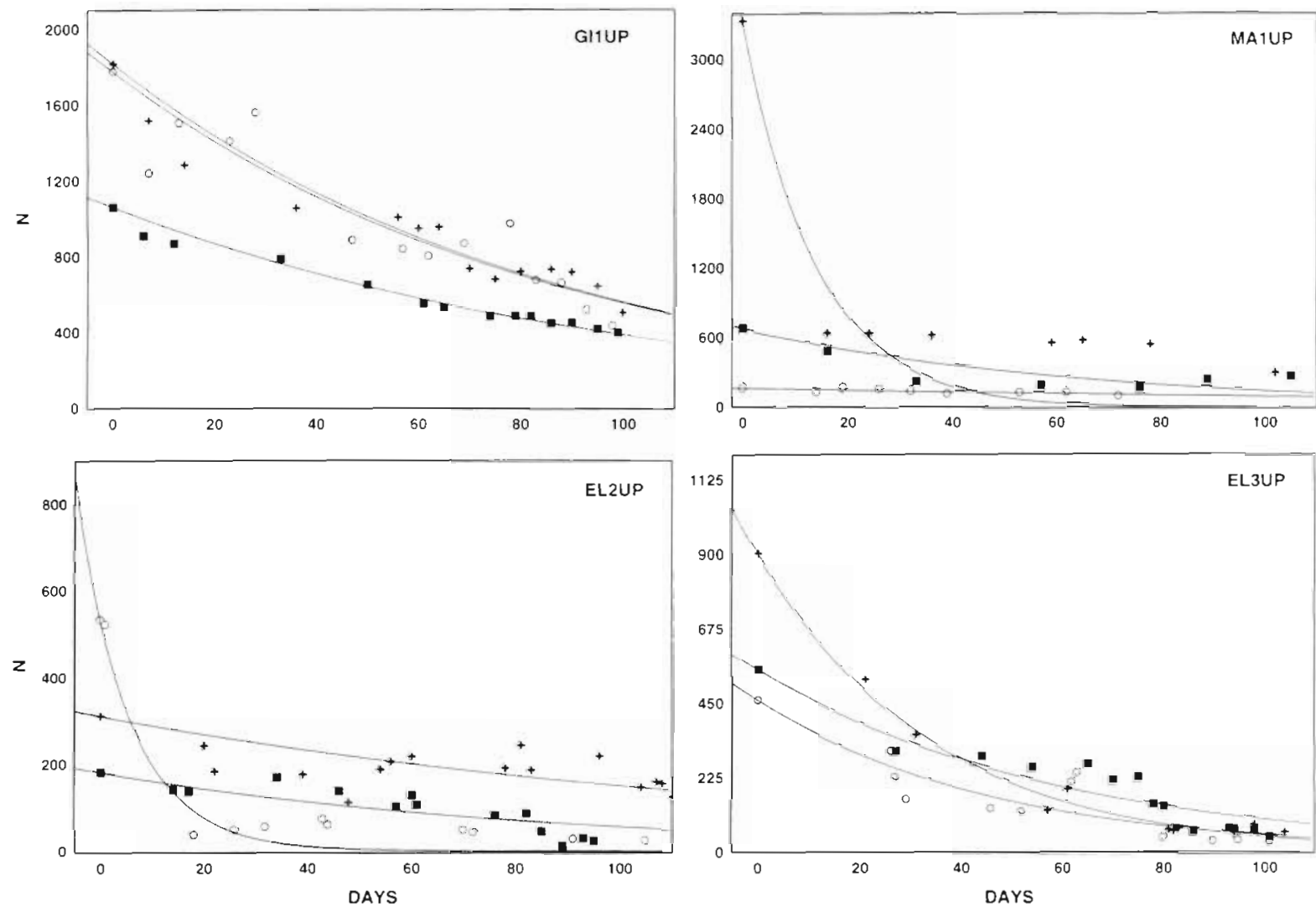

Fig. 4. Diplodus vulgaris. Density time series after peak settlement abundance. N: number of individuals (see text for further explanation). 0: date of maximum abundance at settlement. Years: (0) 1994, (+) 1995, (a) 1996. Station abbreviations as in Fig. 2

ment period. Furthermore, migrations to and from the nursery area need to be accurately assessed in order to avoid bias in the mortality rate estimates (Jones 1991) In addition, this method requires frequent sampling at the time of settlement to obtain an accurate assessment of settlement strength. An inappropriate sampling protocol may fail to reflect the true settlement/post-settlement relationship, thus leading to erroneous conclusions (Booth 1991, Doherty 1991). We consider that the intensive sampling procedure used in the present study, together with the high level of fidelity to the nursery site during the sampling period (Macpherson 1997), can provide an accurate picture of the mortality trends in the 3 species of Diplodus.

Other workers studying juvenile fishes have reported mortality to be either density-dependent or density-independent. In Chromis cyanea, Hixon \& Carr (1997) recently found that in the absence of predators the pattern is density-independent; however, when 2 suites of predators (residents and transiets) are present, mortality is density-dependent. The results of certain studies on coral reef fishes [Pomacentrus wardi and P. flavicauda (Doherty 1982), Pomacentrus amboinensis (Jones 1987), and Thalassoma bifas- ciatum (Victor 1986)] are indicative of density-independent mortality. Conversely, Booth (1995) recorded an increase in the survival of juveniles of Dascyllus albisella with shoal size. Contrasting with the findings of Booth (1995), Eckert (1987) found higher mortality rates in juveniles of species that formed large shoals than among juveniles that formed small aggregations. Other studies on coral reef fishes have shown high inter-annual and inter-site variability in mortality rate estimates (Victor 1986, Eckert 1987, Robertson 1988 , Forrester 1990, Doherty \& Fowler 1994, Booth 1995). Sano (1997) found either a density-dependent or density-independent pattern in Sagamia geneionema in different years. On the other hand, Sale \& Ferrell (1988) found no inter-annual variability in mortality rates, even at differing levels of settlement abundance. In opposition to these findings, results for some species from temperate regions, e.g. flatfishes, gadoids, and. rockfishes, have been indicative of distinctly densitydependent mortality with inter-annual variability in mortality rates, which agrees with the findings of the present study. Mortality on juvenile Pleuronectes platessa has been shown to be clearly density-dependent, though the mortality pattern becomes density- 
Table 4. Average mortality rates (SD in parentheses) for Diplodus puntazzo and $D$. sargus inside the protected areas $(P)$, and in unprotected areas (UP) for 3 levels of maximum settlement abundance (D. vulgaris was not sampled in protected areas), considering sampling period as a whole (1993 to $1995)$ and for each individual year. Differences in mortality rates were tested using the Mann-Whitney $U$-test ( $\mathrm{p}=$ significance level)

\begin{tabular}{|c|c|c|c|}
\hline Abundance & & D. puntazzo & D. sargus \\
\hline $\begin{array}{l}\text { All years } \\
>400 \text { ind. }\end{array}$ & $\begin{array}{l}\text { UP } \\
P\end{array}$ & & $\begin{array}{c}0.026(0.007) \\
0.026(0.007) \\
p=0.35\end{array}$ \\
\hline $100-400$ ind. & $\begin{array}{l}\text { UP } \\
P\end{array}$ & $\begin{array}{c}0.007(0.001) \\
0.009(0.002) \\
p=0.29\end{array}$ & $\begin{array}{c}0.023(0.010) \\
0.017(0.006) \\
p=0.70\end{array}$ \\
\hline$<100$ ind & $\begin{array}{l}\text { UP } \\
P\end{array}$ & $\begin{array}{c}0.005(0.004) \\
0.008(0.002) \\
p=0.12\end{array}$ & $\begin{array}{c}0.011(0.007) \\
0.010(0.003) \\
p=0.40\end{array}$ \\
\hline $\begin{array}{l}1993 \\
<100 \text { ind }\end{array}$ & $\begin{array}{c}\text { UP } \\
P\end{array}$ & $\begin{array}{c}0.006(0.006) \\
0.002(0.002) \\
p=0.24\end{array}$ & \\
\hline $\begin{array}{l}1994 \\
>400 \text { ind. }\end{array}$ & $\begin{array}{c}U P \\
P\end{array}$ & & $\begin{array}{c}0.025(0.014) \\
0.029(0.010) \\
p=0.83\end{array}$ \\
\hline $100-400$ ind. & $\begin{array}{l}\text { UP } \\
P\end{array}$ & & $\begin{array}{c}0.023(0.006) \\
0.024(0.006) \\
p=0.93\end{array}$ \\
\hline$<100$ ind & $\begin{array}{l}\text { UP } \\
P\end{array}$ & $\begin{array}{c}0.004(0.003) \\
0.006(0.003) \\
p=0.15\end{array}$ & \\
\hline $\begin{array}{l}1995 \\
100-400 \text { ind. }\end{array}$ & $\begin{array}{l}\text { UP } \\
P\end{array}$ & & $\begin{array}{c}0.022(0.010) \\
0.015(0.008) \\
p=0.29\end{array}$ \\
\hline$<100$ ind & $\begin{array}{l}\text { UP } \\
P\end{array}$ & & $\begin{array}{c}0.018(0.005) \\
0.004(0.003) \\
p=0.08\end{array}$ \\
\hline
\end{tabular}

independent at very low levels of settlement abundance (Lockwood 1980, van der Veer 1986, Beverton \& Iles 1992b). Myers \& Cadigan (1993a) also reported density-dependent mortality in Gadus morhua, Merlangius merlangus, Pleuronectes platessa, and Solea solea, and Adams \& Howard (1996) observed a similar mortality pattern in Sebastes mystinus.

As has been reported in other species (e.g. Zijlstra et al. 1982, van der Veer 1986, Peterman et al. 1988, Walters \& Collie 1988, Beverton \& Iles 1992b, Bradford 1992, Adams \& Howard 1996), the density-dependent pattern of mortality brought about a reduction in the variability in year-class strength (at least in Diplodus sargus and $D$. puntazzo). The reduction did not change the rank order of abundance between years. Thus, while the magnitude of year-class strength is roughly set before the settlement stage, additional pressure is exerted on juveniles in the early benthic stage. The reduction in variability between year classes acted to smooth out variability in recruitment to the adult population

The wide range of mortality rate values estimated for Diplodus species and the fact that the mortality rate is density-dependent make it necessary to regard interspecific comparisons with caution. In the present study, the 3 species considered displayed high overlap in the mortality rate values, and the only clear differences in mortality rates were between $D$. puntazzo and $D$. sargus and less distinctly between $D$. vulgaris and $D$. sargus. D. puntazzo and $D$. vulgaris settled in the cold part of the year (peak abundance in OctoberNovember and January-February, respectively) and took between 7 and 8 and between 5 and 6 mo, respectively, to grow to the size of 5 to $6 \mathrm{~cm}$ at which they leave the nursery area and recruit to the adult population. D. sargus settled in the warm part of the year (May-June) and needed only 2 or 3 mo to reach a similar size (Macpherson 1997). Mortality rates on D. sargus are generally higher than on $D$. puntazzo and $D$. vulgaris, suggesting that risks are higher in the warmer season.

Protected areas support much higher numbers of large fish predators than unprotected areas do, as a result of the 'reserve effect' (Bell 1983, García-Rubies \& Zabala 1990, Dufour et al. 1995, Harmelin et al. 1995, see also Roberts \& Polunin 1991), suggesting that protected areas might act as a sink for post-settlement fishes. Still, the results of this study indicated that mortality rates on juveniles in protected areas do not differ significantly from those in unprotected areas. At the sampling sites juveniles of Diplodus are preyed upon by many different predators, including cnidarians (Anemonia sulcata), cephalopods (Octopus vulgaris, Sepia officinalis), and more than 15 fish species (e.g. Dicentrarchus labrax, Gobius cobitis, Oblada melanura, Parablennius sanguinolentus, Scorpaena porcus, Serranus scriba, S. cabrilla, Trachinus draco, Tripterygion tripteronotus) (Macperhson et al. unpubl.). The size of the fish predators tends to be small, usually no larger than $20 \mathrm{~cm}$ [new recruits of the sparid Oblada melanura ( 4 to $6 \mathrm{~cm}$ in length) have sometimes been observed to prey on new settlers of Diplodus sargus $(1$ to $2 \mathrm{~cm})]$. An analysis of the impact of predators on juveniles of Diplodus species fell outside the scope of the present study. In any case, small predatory species or small individuals of large species are not seriously affected by the 'reserve effect' and have similar densities in protected and unprotected areas (e.g. Macpherson 1994, Harmelin et al. 1995 , García-Rubies 1997) However, additional studies will 
be required to confirm whether the main cause of mortality on new settlers of Diplodus is predation or another factor (e.g. starvation; Bailey 1994, Leggett \& Deblois 1994).

Acknowledgements. We are very grateful to A. Gordoa for her valuable comments and suggestions. We also thank $\mathrm{V}$. Dufour, R. Galzin, S. Gambaccini, L. LeDireach, L. Mariani, M. Mori, M. Vacchi, and M. Zazzetta for their assistance in collecting data. The manuscript benefits from input from $R$. Sacks. This research was funded by the European Commission (DG XlV, MED/92/007)

\section{LITERATURE CITED}

Adams PB, Howard DF (1996) Natural mortality of blue rockfish, Sebastes mystinus, during their first year in nearshore benthic habitats. Fish Bull 94:156-162

Bailey KM (1994) Predation on juvenile flatfish and recruitment variability. Neth J Sea Res 32:175-189

Bailey KM, Houde ED (1989) Predation on eggs and larvae of marine fishes and the recruitment problem. Adv Mar Biol 25:1-81

Bell JD (1983) Effects of depth and marine reserve fishing restrictions on the structure of a rocky reef fish assemblage in the North-Western Mediterranean Sea. J Appl Ecol 20:357-369

Beverton RJH, Iles TC (1992a) Mortality rates of 0-group plaice (Pleuronectes platessa L.), dab (Limanda limanda L.) and turbot (Scophthalmus maximus L.) in European waters. II. Comparison of mortality rates and construction of life table for 0-group plaice. Neth $J$ Sea Res 29:49-59

Beverton RJH, Iles TC (1992b) Mortality rates of 0-group plaice Pleuronectes platessa L., dab Limanda limanda L. and turbot Scophthalmus maximus L. in European waters. III. Density-dependence of mortality rates of 0 -group plaice and some demographic implications. Neth J Sea Res 29:61-79

Bohnsack JA (1993) Marine reserves: they enhance fisheries, reduce conflicts, and protect resources. Oceanus 36:63-71

Booth DJ (1991) The effects of sampling frequency on estimates of recruitement of the domino damselfish Dascyllus albisella Gill. J Exp Mar Biol Ecol 145:149-159

Booth DJ (1995) Juvenile groups in a coral-reef damselfish: density-dependent effects on individual fitness and population demography. Ecology 76:91-106

Booth DJ, Beretta GA (1994) Seasonal recruitment, habitat associations and survival of pomacentrid reef fish in the US Virgin Islands. Coral Reefs 13:81-89

Bradford MJ (1992) Precision of recruitment predictions from early life stages of marine fishes. Fish Bull 90:439-453

Caley MJ, Carr MH, Hixon MA, Hughes TP, Jones GP, Menge BA (1996) Recruitment and the local dynamics of open marine populations. Annu Rev Ecol Syst 27:477-500

Carr $M H$, Hixon MA (1995) Predation effects on early postsettlement survivorship of coral-reef fishes. Mar Ecol Prog Ser 124:31-42

Carr MH, Reed DC (1993) Conceptual issues relevant to marine harvest refuges: examples from temperate reef fishes. Can J Fish Aquat Sci 50:2019-2028

Connell SD (1997) The relationship between large predatory fish and recruitment and mortality of juvenile coral reeffish on artificial reefs. J Exp Mar Biol Ecol 209:261-278

Cushing DH (1977) The problems of stock and recruitment. In: Gulland JA (ed) Fish population dynamics. Wiley,
Toronto, p 1.16-135

Dempster JP, Pollard E (1986) Spatial heterogeneity, stochasticity and the detection of density dependence in anumal populations. Oikos 46:413-416

Dennis B, Taper ML (1994) Density dependence in time series observations of natural populations: estimation and testing. Ecol Monogr 64:205-224

Doherty PJ (1982) Some effects of density on the juveniles of two species of tropical, territorial damselfishes. J Exp Mar Biol Ecol 65:249-261

Doherty PJ (1991) Spatial and temperature patterns in recruutment. In: Sale PF (ed) The ecology of fishes on coral reefs. Academic Press, London, p 261-292

Doherty PJ, Fowler T (1994) An empirical test of recruitment limitation in a coral reef fish. Science 263:935-939

Doherty PJ, Williams DMcB (1988) The replenishment of coral reef fish populations. Oceanogr Mar Biol Annu Rev 26: $487-551$

Dufour V, Jouvenel JY, Galzin R (1995) Study of Mediterranean reef fish assemblage. Comparison in population distribution among depth in protected and unprotected areas over one decade. Aquat Living Resour 8:17-25

Eckert GJ (1987) Estimates of adult and juvenile mortality for labrid fishes at One Tree Reef, Great Barrier Reef. Mar. Biol 95:167-171

Ellis T, Gibson RN (1995) Size-selective predation of 0-group flatfishes on a Scottish coastal nursery ground. Mar Ecol Prog Ser 127:27-37

Forrester GE (1990) Factors influencing the juvenile demography of a coral reef fish population. Ecology 71 : $1666-1681$

García-Rubies A (1997) Estudi ecològic de les poblacions de peixos litorals sobre substrat rocòs a la Mediterrània occidental: efecte de la fondària, el substrat, l'estacionalitat y la pretecció. PhD thesis, Univ Barcelona

García-Rubies A, Macpherson E (1995) Substrate use and temporal pattern of recruitment in juvenile fishes of the Mediterranean littoral. Mar Biol 124:35-42

Garcia-Rubies A, Zabala M (1990) Effects of total fishing prohibition on the rocky fish assemblages of Medes island marine reserve NW Mediterranean. Sci Mar 544:317-328

Gaston KJ, Lawton JH (1987) A test of statistical techniques for detecting density dependence in sequential censuses of animal populations. Oecologia 74:404-410

Hanski I (1990) Density dependence, regulation and variability in animal populations. In: Hassell MP, May RM (eds) Population regulation and dynamics. Phil Trans R Soc Lond Ser B 330:141-150

Hanski I, Woiwod I, Perry J (1993) Density dependence, population persistence and largely futile arguments. Oecologia 95:595-598

Harmelin JG (1987) Structure et variabilité de l'ichtyofaune d'une zone rocheuse protégée en Méditerranée Parc national de Port-Cros, France. PSZN I: Mar Ecol 83: $263-284$

Harmelin JG, Bachet F, Garcia F (1995) Mediterranean marine reserves: fish indices as tests of protection efficiency. PSZN I: Mar Ecol 16:233-250

Harmelin-Vivien ML, Harmelin JG, Leboulleux F (1995) Microhabitat requirements for settlement of juvenile sparid fishes on Mediterranean rocky shores. Hydrobiologia 300/301:309-320

Hassell MP (1986) Detecting density dependence. Trends Ecol Evol 1:90-93

Hixon MA, Carr MH (1997) Synergistic predation, density dependence, and population regulation in marine fish Science 277:946-949 
Iles TC, Beverton RJH (1991) Mortality rates of 0-group plaice Pleuronectes platessa $\mathrm{L}$. dab Limanda limanda $\mathrm{L}$. and turbot Scophthalmus maximus L. in European waters. I. Statistical analysis of the data and estimation of parameters. Neth J Sea Res 27:217-235

Jager Z, Kleef HL, Tydeman P (1995) Mortality and growth of 0-group flatfish in the brackish Dollard (Ems estuary, Wadden Seal. Neth J Sea Res 34:119-129

Jones GP (1987) Some interactions between residents and recruits in two coral reef fishes. J Exp Mar Biol Ecol 114 $169-182$

Jones GP (1991) Postrecruitment processes in the ecology of coral reef fish populations: a multifactorial perspective. In Sale PF (ed) The ecology of fishes on coral reefs. Academic Press, London. p $294-327$

Leggett WC, Deblois E (1994) Recruitment in marine fishes: is it regulated by starvation and predation in the egg and larval stages. Neth J Sea Res 32:119-134

Levin PS (1994) Fine-scale temporal variation in recruitment of a temperate demersal fish: the importance of settlement versus post-settlement loss. Oecologia 97:124-133

Lockwood SJ (1980) Density-dependent mortality in 0-group plaice Pleuronectes platessa L. populations. J Cons Int Explor Mer 39:148-153

Macpherson E (1994) Substrate utilisation in a Mediterranean littoral fish community. Mar Ecol Prog Ser 114:211-218

Macpherson E (1997) Ontogenetic shifts in habitat use and aggregation in juvenile sparid fishes. J Exp Mar Biol Ecol (in press)

Myers RA, Cadigan NG (1993a) Density-dependent juvenile mortality in marine demersal fish. Can J Fish Aquat Sci 50 $1576-1590$

Myers RA, Cadigan NG (1993b) Is juvenile natural mortality in marine demersal fish variable? Can J Fish Aquat Sci 50: $1591-1598$

Peterman RM, Bradford MJ, Lo NCH, Methot RD (1988) Contribution of early life stages to interannual variability in recruitment of northern anchovy Engraulis mordax. Can J Fish Aquat Sci 45:8-16

Roberts CM, Polunin NVC (1991) Are marine reserves effective in management of reef fisheries? Rev Fish Biol Fish 1. $65-91$

Robertson DR (1988) Abundances of surgeonfishes on patchreefs in Caribbean Panama: due to settlement, or postsettlement events? Mar Biol 97:495-501

Sala E. Boudouresque CF (1997) The role of fishes in the organization of a Mediterranean sublittoral community. I. Algal communities. J Exp Mar Biol Ecol 212:25-44

Sala E, Zabala M (1996) Fish predation and the structure of the sea urchin Paracentrotus lividus populations in the NW Mediterranean. Mar Ecol Prog Ser 140:71-81

Sale PF, Ferrell DJ (1988) Early survivorship of juvenile coral

Editorial responsibility: Otto Kinne (Editor)

Oldendorf/Luhe, Germany reef fishes. Coral Reefs 7:117-124

Sano $M$ (1997) Temporal variation in density dependence: recruitment and postrecruitment demography of a temperate zone sand goby. J Exp Mar Biol Ecol 214:67-84

Shepherd JG, Cushing DH (1990) Regulation in fish population: myth or mirage. Phil Trans R Soc Lond B 330:151-164

Shulman MJ, Ogden JC (1987) What controls tropical reef fish populations: recruitment or benthic mortality? An example in the Caribbean reef fish Haemulon flavolineatum. Mar Ecol Prog Ser 39:233-242

Steele MA (1997) The relative importance of processes affecting recruitment of two temperate reef fishes. Ecology 78 $129-146$

Sundby S, Bjorke H, Soldal AV, Olsen S (1989) Mortality rates during the early life stages and year-class strength of northeast Arctic cod Gadus morhua L. Rapp PV Reun Cons Perm lnt Explor Mer 191:351-358

Tanaka M, Goto T, Tomiyama M, Sudo H (1989) Immigration, settlement and mortality of flounder Paralichthys olivaceus larvae and juveniles in a nursery ground, Shijiki Bay, Japan. Neth J Sea Res 24:57-67

Tupper M, Boutilier RG (1995) Effects of habitat on settlement, growth, and postsettlement survival of Atlantic cod Gadus morhua. Can J Fish Aquat Sci 52:1834-1841

van der Veer HW (1986) Immigration, settlement and densitydependent mortality of a larval and and early post-larval o-group plaice Pleuronectes platessa population in the western Wadden Sea. Mar Ecol Prog Ser 29:223-236

van der Veer HW, Berghahn MJN (1987) Predation by crustaceans on a newly settled 0-group plaice Pleuronectes platessa population in the western Wadden Sea. Neth J Sea Res 35:203-215

van der Veer HW, Berghahn MJN, Dapper R, Witte JIJ (1991) Population dynamics of an intertidal 0-group flounder Platichthys flesus population in the western Dutch Wadden Sea. Mar Ecol Prog Ser 73:141-148

Vetter EF (1988) Estimation of natural mortality in fish stocks: a review. Fish Bull 86:25-43

Victor BC (1986) Larval settlement and juvenile mortality in a recruitment-limited coral reef fish population. Ecol Monogr 56:145-160

Walters CJ, Collie JS (1988) Is research on environmental factors useful to fisheries management? Can J Fish Aquat Sci 45:1848-1854

Williams DMcB, Engish W, Milicich MJ (1994) Annual recruitment surveys of coral reef fishes are good indicators of patterns of settlement. Bull Mar Sci 54:314-331

Zar JH (1984) Biostatistical analysis. Prentice-Hall International, Englewood Cliffs, NJ

Zijlstra JJ, Dapper R, Witte JIJ (1982) Settlement, growth and mortality of post-larval plaice Pleuronectes platessa in the Western Wadden Sea. Neth J Sea Res 15:250-272

Submitted: May 16, 1997; Accepted: October 14, 1997

Proofs received from author(s): December 8, 1997 